\title{
Artificial Neural Network Models based Cardiac Arrhythmia Disease Diagnosis from ECG Signal Data
}

\author{
Shivajirao M. Jadhav \\ Dr. Babasaheb Ambedkar \\ Technological University \\ Lonere 402103 Dist. Raigad \\ M.S. India
}

\author{
Sanjay L. Nalbalwar \\ Dr. Babasaheb Ambedkar \\ Technological University \\ Lonere 402103 Dist. Raigad \\ M.S. India
}

\author{
Ashok A. Ghatol \\ K. J. Educational Institutes \\ Pisoli, Kondhwa-Saswad Road, \\ Near Bopdeo Ghat, District \\ Pune PIN-411 048, M.S. India
}

\begin{abstract}
Changes in the normal rhythm of a human heart may result in different cardiac arrhythmias, which may be immediately causes irreparable damage to the heart sustained over long periods of time. The ability to automatically identify arrhythmias from ECG recordings is important for clinical diagnosis and treatment. In this paper we proposed an Artificial Neural Network (ANN) based cardiac arrhythmia disease diagnosis system using standard 12 lead ECG signal recordings data. In this study, we are mainly interested in classifying disease in normal and abnormal classes. We have used UCI ECG signal data to train and test three different ANN models. In arrhythmia analysis, it is unavoidable that some attribute values of a person would be missing. Therefore we have replaced these missing attributes by closest column value of the concern class. ANN models are trained by static backpropagation algorithm with momentum learning rule to diagnose cardiac arrhythmia. The classification performance is evaluated using measures such as mean squared error (MSE), classification specificity, sensitivity, accuracy, receiver operating characteristics (ROC) and area under curve (AUC). Out of three different ANN models Multilayer perceptron ANN model have given very attractive classification results in terms of classification accuracy and sensitivity of $86.67 \%$ and $93.75 \%$ respectively while Modular ANN have given $93.1 \%$ classification specificity.
\end{abstract}

\section{General Terms}

Machine Learning, Pattern Classification.

\section{Keywords}

Accuracy, ECG Arrhythmia, Multilayer perceptron neural network model, momentum learning rule, sensitivity, specificity

\section{INTRODUCTION}

The cardiovascular disease becomes one of main diseases that threaten the human especially in developing countries such USA and Canada. There are many ways to discover cardiac arrhythmias by using ECG signal. Electrocardiography is important device for recording electrocardiogram (ECG) signals and variability of bioelectric potential with respect to time as human heart beats. ECG is widely used for diagnosing heart activity among three features known as $\mathrm{P}$, QRS and T waves. Also it gives us useful information about the functional aspect of the heart. The early detection of the cardiac arrhythmias can prolong life and enhance the quality of living through appreciates treatment. Therefore, we need many techniques that analyze the ECG signal to detect the heart diseases. The state of cardiac health is generally reflected in the shape of the ECG waveform and heart rate and contains important pointers to nature of the disease attacking the heart [1].

Electrocardiogram records the electronic activities of the heart, and has been widely adapted for diagnosing cardiac arrhythmia [2]. Approaches have already been developed for classifying cardiac arrhythmias based on ECG signal but still weak and have a poor accuracy because they depend on features extraction of ECG in order to classify cardiac arrhythmias. Various Machine learning and data mining methods have been applied to improve the accuracy for the detection of ECG arrhythmia.

\section{RELATED REASEARCH WORK}

Automatic ECG analysis is critical for diagnosis and treatment of critically ill patients. Modeling and simulation of ECG under various conditions are very important in understanding the functioning of the cardiovascular system as well as in the diagnosis of heart diseases. Arrhythmias represent a serious threat to the patient recovering from acute myocardial infarction, especially ventricular arrhythmias like ventricular tachycardia (VT) and ventricular fibrillation (VF) [3]. In the present scenario, cardiovascular diseases have proved to be one of the major causes of casualties. Arrhythmia is defined as any sort of disorder that takes place in normal rhythm of heart. Some arrhythmias like ventricular fibrillation are fatal and also can cause death of patient. The classification of arrhythmias is very important as some arrhythmias are severely fatal while others are not. The detection of arrhythmia is an important task in clinical reasons which can initiate life saving operations [4]. Several methods for automated arrhythmia detection have been developed in the past few decades to attempt simplify the monitoring task [5]. These include Wavelet transformation [6-8], Radial Basis Functions (RBF) Neural Networks [9], self- organizing map [10] and fuzzy c-means clustering techniques [11]. Multilayer neural networks have also been used to classify arrhythmia QRS complexes, and for ischemia detection [12-13]. Partial parts of this works are available in [14-17]. An Artificial immune recognition system (AIRS) with fuzzy weighted preprocessing [18] is also used for arrhythmia classification. Multilayer perceptron model and Modular neural network model is applied in [19] and [20] respectively for multiclass ECG arrhythmia and fetal state classification problems. Various neural network models are used to classify ECG arrhythmia and classification accuracies are reported in [2124].

In this paper, we have considered three different neural network models to classify arrhythmia cases into normal and abnormal. Three different ANN models designed are Multilayer Perceptron (MLP), Generalized Feedforward Neural Network (GFFNN) model and Modular Neural 
Network (MNN) model. The performances of experimental results of these models are measured using standard classifier measures such as accuracy, sensitivity, specificity and area under receiver operating characteristics. All network models are trained with static backpropagation algorithm with momentum learning rule. The proposed method first cleans the data set by replacing missing values by closest column values of the concern class. The data set used for experimental purpose is the UCI cardiac arrhythmia ECG signal recordings data which contains 452 instances with 279 attributes [25].

\section{NEURAL NETWORKS MODELS USED}

\subsection{Multilayer Perception Neural Network Model}

ANNs is a data processing mathematical model. It consists of a number of units or elements called nodes or neurons. These nodes or neurons are arranged in layers and are interconnected by weights and biases between the layers. It is one of the most widely implemented neural network topologies. MLP is just that, a network that is comprised of many neurons, divided in layers. These layers are input layer, one or more hidden layer and output layer. Lippman [26] is probably one of the best references for the computational capabilities of MLPs.

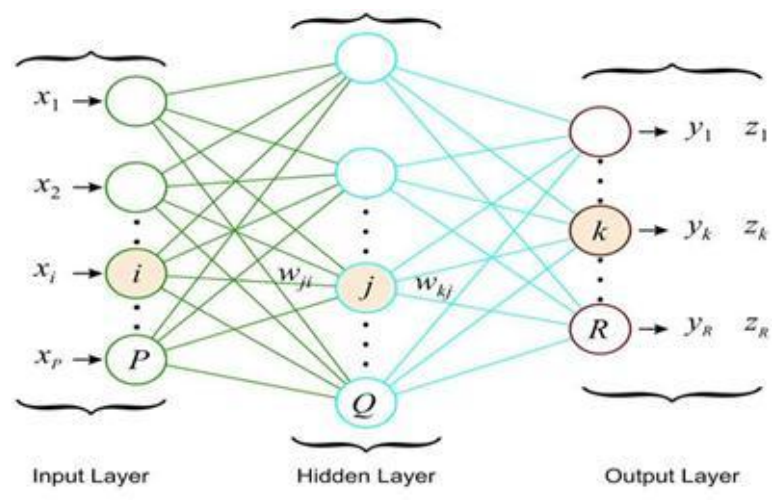

Fig 1: Multilayer Perceptron Model

\subsection{Generalized Feed forward Neural \\ Network Model}

Generalized feedforward neural networks are a generalization of the Multilayer perceptron (MLP) such that connections can jump over one or more layers. In theory, a MLP can solve any problem that a generalized feedforward network can solve. In practice, however, generalized feedforward networks often solve the problem much more efficiently. A classic example of this is the two spiral problem. Without describing the problem, it suffices to say that a standard MLP requires hundreds of times more training epochs than the generalized feedforward network containing the same number of processing elements.

\subsection{Modular Neural Network Model}

A modular neural network (MNN) is a neural network characterized by a series of independent neural networks moderated by some intermediary. Each independent neural network serves as a module and operates on separate inputs to accomplish some subtask of the task the network hopes to perform. The intermediary takes the outputs of each module and processes them to produce the output of the network as a whole. MNNs are normally trained with the backpropagation algorithm.

\section{METHODS}

\subsection{Problems and Data Sets:}

The dataset used in this study was obtained from the archives of UCI machine-learning repository datasets [25]. In the data set, Class 01 refers to 'normal' ECG. Classes 02-15 refer to different classes of arrhythmia and Class 16 refers to the rest of unclassified data. The arrhythmia dataset has 279 attributes, 206 of which are linear valued and the rest are nominal. There are 452 instances, and as indicated above, 16 classes. There are missing values in the dataset. In such cases, a closest column value of concern class is used to replace theses missing values.

The original data set grouped into different data sets as shown in the Table 1 and each group is partitioned into two subsets viz. training set and testing set except the last group in which all 452 instances are used for training purpose only.

Table 1: Data Set Group Partitions

\begin{tabular}{|l|l|l|l|l|}
\hline $\begin{array}{l}\text { Data } \\
\text { Set }\end{array}$ & $\begin{array}{l}\text { Training } \\
\% \text { age }\end{array}$ & $\begin{array}{l}\text { Testing } \\
\% \text { age }\end{array}$ & $\begin{array}{l}\text { Training } \\
\text { instances }\end{array}$ & $\begin{array}{l}\text { Testing } \\
\text { Instances }\end{array}$ \\
\hline $\begin{array}{l}\text { Data set } \\
\text { (DS1) }\end{array}$ & 80 & 20 & 362 & 90 \\
\hline $\begin{array}{l}\text { Data set } \\
\text { (DS2) }\end{array}$ & 75 & 25 & 339 & 113 \\
\hline $\begin{array}{l}\text { Data set } \\
\text { 3 (DS3) }\end{array}$ & 70 & 30 & 316 & 136 \\
\hline $\begin{array}{l}\text { Data set } \\
\text { 4 (DS4) }\end{array}$ & 85 & 15 & 384 & 68 \\
\hline $\begin{array}{l}\text { Main } \\
\text { Dataset }\end{array}$ & $\begin{array}{l}\text { Basic Data set itself (all } 452 \text { instances are used } \\
\text { for training only) }\end{array}$ \\
\hline
\end{tabular}

\subsection{Back propagation algorithm}

The back propagation learning algorithm uses the deltarule. What this does is that it computes the deltas, (local gradients) of each neuron starting from the output neurons and going backwards until it reaches the input layer. To compute the deltas of the output neurons, we first have to get the error of each output neuron. Thus backpropagation rule propagates the errors through the network and allows adaptation of the hidden processing elements (PEs). Two important characteristics of the multilayer perceptron are: its nonlinear processing elements (PEs) which have a nonlinearity that must be smooth (the logistic function and the hyperbolic tangent are the most widely used); and their massive interconnectivity (i.e. any element of a given layer feeds all the elements of the next layer). The multilayer perceptron is trained with error correction learning, which means that the desired response for the system must be known. In pattern recognition this is normally the case, since we have our input data labeled, i.e. we know which data belongs to which experiment.

Error correction learning works in the following way: From the system response at $P E_{i}$ at iteration $n, y_{i}(n)$ and the desired response $d_{i}(n)$ for a given input pattern an instantaneous error, $e_{i}(n)$ is defined by:

$$
e_{i}(n)=a_{i}(n)-y_{i}(n)
$$


Using the theory of gradient descent learning, each weight in the network can be adapted by correcting the present value of the weight with a term that is proportional to the present input and error at the weight, i.e.

$$
w_{i i}(n+1)=w_{i i}(n)+\eta \delta_{i}(n) x_{j}(n)
$$

The local error $\delta_{i}(n)$ can be directly computed from $e_{i}(n)$ at the output PE or can be computed as a weighted sum of errors at the internal PEs. The constant $\eta$ is called the step size. This procedure is called the backpropagation algorithm. Backpropagation computes the sensitivity of a cost functional with respect to each weight in the network, and updates each weight proportional to the sensitivity. The beauty of the procedure is that it can be implemented with local information and requires just a few multiplications per weight, which is very efficient. Because this is a gradient descent procedure, it only uses the local information so can be caught in local minima. Moreover, the procedure is inherently noisy since we are using a poor estimate of the gradient, causing slow convergence. Momentum learning is an improvement to the straight gradient descent in the sense that a memory term (the past increment to the weight) is used to speed up and stabilize convergence. In momentum learning the equation to update the weights becomes:

$$
\begin{aligned}
w_{i j}(n+1)=w_{i j}(n) & +\eta \delta_{i}(n) x_{j}(n) \\
+ & \alpha\left(w_{i j}(n)-w_{i j}(n-1)\right)
\end{aligned}
$$

Where $\alpha$, is the momentum and normally it should be set between 0.1 and 0.9 [27].

\subsection{Performance Measures}

We have evaluated the performance of the classification algorithms using six measures; sensitivity, specificity, classification accuracy, mean squared error (MSE), receiver operating characteristics (ROC) and area under ROC curve (AUC). These measures are defined using True Positive (TP), True Negative (TN), False Positive (FP) and False Negative (FN). TP decision occurs when an arrhythmia detection of the classifier coincided with a decision of the physician. TN decision occurs when both the classifier and the physician suggested the absence of arrhythmia. FP occurs when the system labels a healthy case as an arrhythmia one. Finally, FN occurs when the system labels an arrhythmia case as healthy.

\subsubsection{Classification Accuracy}

Classification accuracy is defined as the ratio of the number of correctly classified cases and is equal to the sum of TP and $\mathrm{TN}$ divided by the total number of cases $\mathrm{N}$.

$$
\text { Accuracy }=(T P+T N) / N
$$

\subsubsection{Classification Sensitivity}

Sensitivity refers to the rate of correctly classified positive and is equal to TP divided by the sum of TP and FN. Sensitivity may be referred as a True Positive Rate.

$$
\text { Sensitivity }=T P /(T P+F N)
$$

\subsubsection{Classification Specificity}

Specificity refers to the rate of correctly classified negative and is equal to the ratio of TN to the sum of TN and FP. False Positive Rate equals (100-specificity).

$$
\text { Specificity }=T N /(F P+T N)
$$

\subsubsection{Mean Squared Error (MSE)}

The mean squared error is simply two times the average cost. The formula for the mean squared error is:

$$
M S E=\frac{\sum_{j=0}^{P} \sum_{i=0}^{N}\left(d_{i j}-y_{i j}\right)^{2}}{N P}
$$

Where,

$$
\begin{aligned}
& P=\text { number of output processing elements (PEs) } \\
& N=\text { number of exemplars (instances) in the data set } \\
& y_{i j}=\text { Network output for exemplar i at processing } \\
& \text { element } \mathrm{j} \\
& d_{i j}=\text { desired output for exemplar i at processing } \\
& \text { element } \mathrm{j}
\end{aligned}
$$

\subsubsection{Area under ROC Curve (AUC)}

AUC has been recently used as an alternative measure for machine learning algorithms. AUC has many advantages such as its independence to the decision sensitivity in analysis of variance (a collection of statistical models and their associated procedures which compare means by splitting the overall observed variance into different parts) tests, its independence to the decision threshold, and its invariance to a priori class probability (recognized in advance as equally probable) distribution etc. Since the AUC is a portion of the area of the unit square, its value will always be between 0 and 1 [28]. Receiver Operating Characteristic (ROC) analysis originated in electrical engineering in the early 1950's where the technique was developed to assess the performance of signal detection devices (receivers). From there it spread into other fields, finding useful applications in both psychology and medical diagnosis. The receiver constantly sees small amounts of noise, so a threshold must be set to distinguish between an actual signal and background noise. Anything below the threshold will be classified as "noise", while anything above the threshold will be classified as "signal". ROC matrices are used to show how changing the detection threshold affects detections versus false alarms. If the threshold is set too high then the system will miss too much detection. Conversely, if the threshold is set too low then there will be too many false alarms [29]

\section{EXPERIMENTS AND RESULTS}

In this study, we have performed experiments on each individual dataset groups using Neurosolutions for Excel software tool [30] using three different ANN topologies. For each network topology (model), we have varied one to three numbers of hidden layers. Table 2 gives cardiac arrhythmia classification results in terms of accuracy, specificity and sensitivity for three different neural network models. Table 3 gives comparison our work (MNN, GFFNN and MLP) models classification accuracy and classification accuracies 
obtained by other methods in literature for ECG Arrhythmia classification problem. Overall training was very good for almost all network models. Training results are very attractive for all network design models on DSMains data set for which $100 \%$ data is used for training purpose only. Average MSE (training) for various network models is shown figure 2, here MLP model have given best training MSE. Testing accuracy is very attractive for MLP network on data set DS5 and it found to be $86.67 \%$ as shown in figure 3 . Classification sensitivity is also very good and it is $93.75 \%$ for data set DS5 on MLP network model with two numbers of Hidden layers and this is shown in figure 4. MLP have given better performance in terms of MSE, accuracy and sensitivity however specificity given by MLP network model is poor as compared with GFNN and MNN models as shown in figure 5. The Modular neural network model with one number of hidden layer have given better classification specificity on data set 5 and it is $93.1 \%$. From table 2 it is clear that, DS4 have given better results on GFFN model with two numbers of hidden layers while DS5 have given best results on MLP and MNN models.

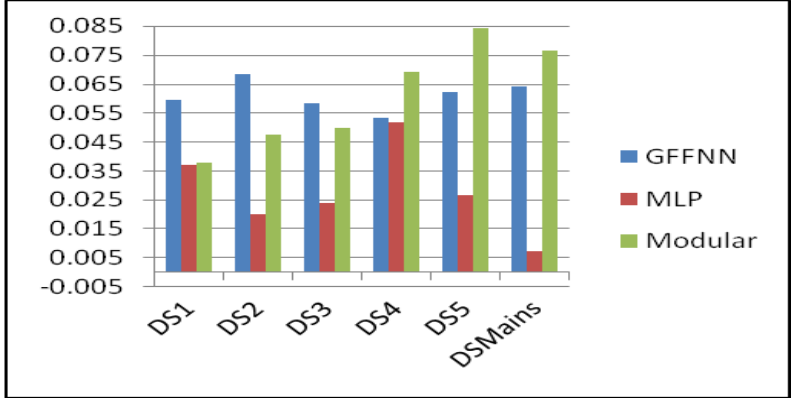

Fig 2: Average Training MSE for three models

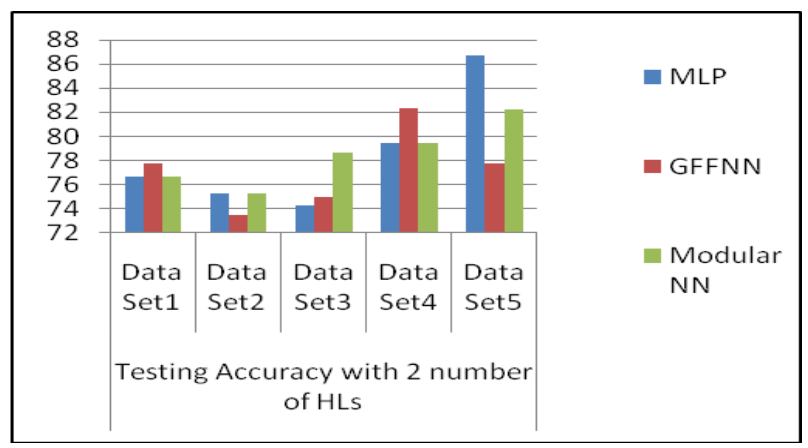

Fig 3: Testing accuracy for all data sets

Table 2. Cardiac Arrhythmia Classification Results

\begin{tabular}{|c|c|c|c|c|c|c|c|c|c|c|}
\hline \multirow{2}{*}{ Data Set } & \multirow{2}{*}{$\begin{array}{c}\text { Number } \\
\text { of HLs }\end{array}$} & \multicolumn{3}{|c|}{ MNN Model } & \multicolumn{3}{|c|}{ GFNN Model } & \multicolumn{3}{|c|}{ MLP Model } \\
\hline & & Sensitivity & Specificity & Accuracy & Sensitivity & Specificity & Accuracy & Sensitivity & Specificity & Accuracy \\
\hline \multirow[b]{3}{*}{ DS1 } & 1 & 73.68 & 82.69 & 78.89 & 73.68 & 80.77 & 77.78 & 71.05 & 86.54 & 80 \\
\hline & 2 & 65.79 & 84.62 & 76.67 & 65.79 & 86.54 & 77.78 & 68.42 & 82.69 & 76.67 \\
\hline & 3 & 71.05 & 84.62 & 78.89 & 60.53 & 84.62 & 74.44 & 71.05 & 84.62 & 78.89 \\
\hline \multirow[b]{3}{*}{ DS2 } & 1 & 68.63 & 82.26 & 76.11 & 76.47 & 83.87 & 80.53 & 66.67 & 83.87 & 76.11 \\
\hline & 2 & 70.59 & 79.03 & 75.22 & 68.63 & 77.42 & 73.45 & 64.71 & 83.87 & 75.22 \\
\hline & 3 & 62.75 & 88.71 & 76.99 & 60.78 & 87.1 & 75.22 & 68.63 & 82.26 & 76.11 \\
\hline \multirow[b]{3}{*}{ DS3 } & 1 & 67.19 & 76.39 & 72.06 & 67.19 & 80.56 & 74.26 & 60.94 & 80.56 & 71.32 \\
\hline & 2 & 73.44 & 83.33 & 78.68 & 68.75 & 80.56 & 75 & 65.63 & 81.94 & 74.26 \\
\hline & 3 & 64.06 & 79.17 & 72.06 & 64.06 & 84.72 & 75 & 65.63 & 79.17 & 72.79 \\
\hline \multirow[b]{3}{*}{ DS4 } & 1 & 65.63 & 83.33 & 75 & 68.18 & 82.61 & 77.94 & 75 & 83.33 & 79.41 \\
\hline & 2 & 75 & 83.33 & 79.41 & 68.18 & 89.13 & 82.35 & 68.18 & 84.78 & 79.41 \\
\hline & 3 & 84.38 & 77.78 & 80.88 & 72.73 & 80.43 & 77.94 & 68.18 & 86.96 & 80.88 \\
\hline \multirow[b]{3}{*}{ DS5 } & 1 & 62.5 & 93.1 & 82.22 & 60 & 88 & 75.56 & 81.25 & 86.21 & 84.44 \\
\hline & 2 & 81.25 & 82.76 & 82.22 & 75 & 80 & 77.78 & 93.75 & 82.76 & 86.67 \\
\hline & 3 & 75 & 85.71 & 80 & 60 & 92 & 77.78 & 81.25 & 79.31 & 80 \\
\hline \multirow[b]{3}{*}{ DSMains } & 1 & 97.89 & 94.85 & 96.32 & 97.82 & 92.26 & 94.87 & 100 & 99.52 & 99.76 \\
\hline & 2 & 99.15 & 94.91 & 96.94 & 98.69 & 92.74 & 95.52 & 100 & 99.52 & 99.76 \\
\hline & 3 & 99.54 & 89.57 & 93.95 & 98.71 & 94.02 & 96.25 & 99.59 & 100 & 99.8 \\
\hline
\end{tabular}

Table 3. Our Work (MNN, GFNN and MLP Models) classification accuracy for ECG Arrhythmia Classification Problem With classification accuracies obtained by other methods in literature

\begin{tabular}{|l|l|c|c|c|c|c|c|c|}
\hline NEWFM & HLVQ & KDFW- & Fuzzy weighted & SVM with & A novel & \multicolumn{3}{|c|}{ (Our work) } \\
[21] & {$[22]$} & KNN [2] & AIRS [18] & $\begin{array}{l}\text { Gaussian } \\
\text { Kernel [23] }\end{array}$ & $\begin{array}{l}\text { pruning } \\
\text { approach [24] }\end{array}$ & $\begin{array}{c}\text { MNN } \\
\text { Model }\end{array}$ & $\begin{array}{c}\text { GFFNN } \\
\text { Model }\end{array}$ & $\begin{array}{c}\text { MLP } \\
\text { Model }\end{array}$ \\
\hline 81.32 & 76.92 & 70.66 & 80.71 & 76.1 & 68.47 & $\mathbf{8 2 . 2 2 \%}$ & $\mathbf{8 2 . 3 5 \%}$ & $\mathbf{8 6 . 6 7 \%}$ \\
\hline
\end{tabular}




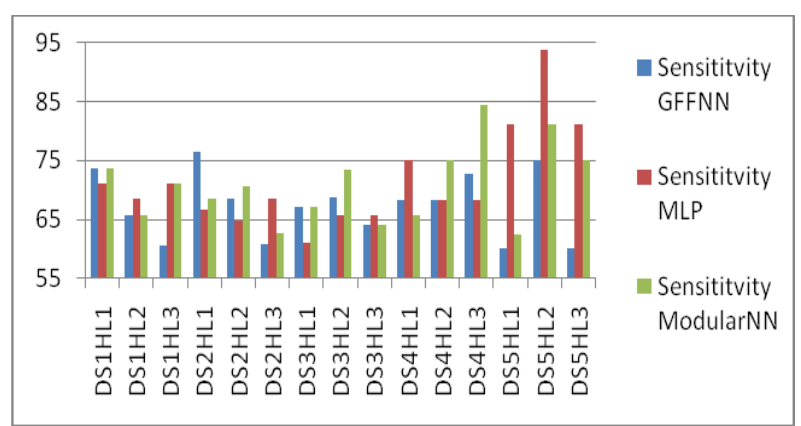

Fig 4: Testing Sensitivity for all data sets

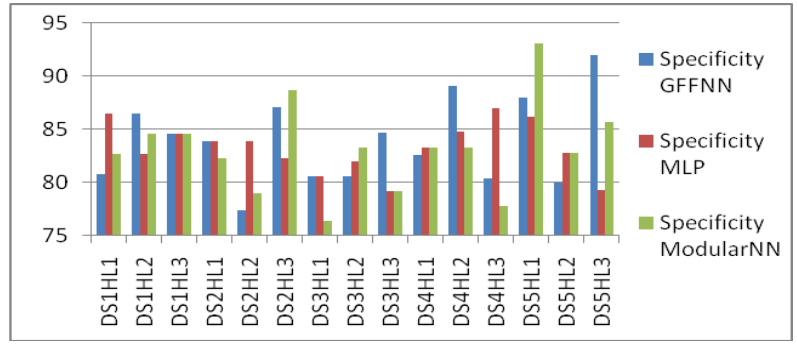

Fig 5: Testing Specificity for all data sets

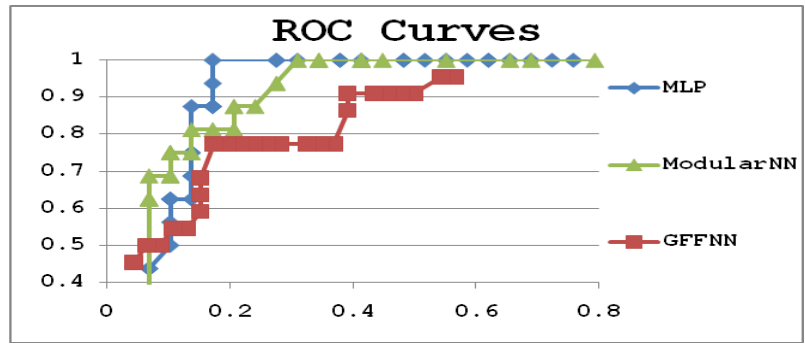

Fig 6: ROC Curves

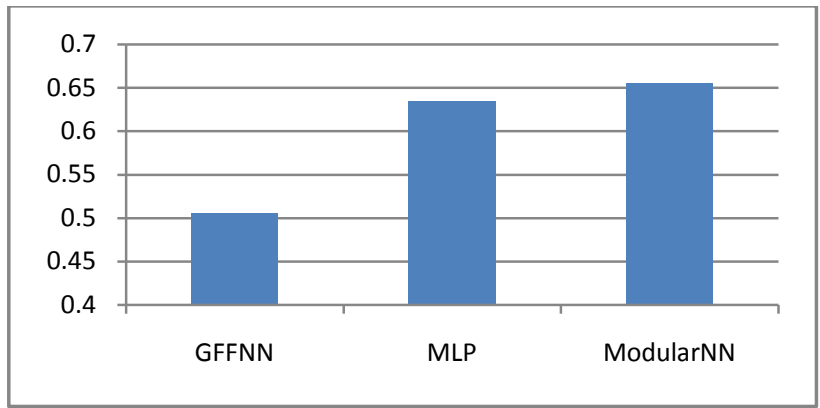

Fig 7: Area under ROC Curves

Therefore receivers operating characteristic curves are drawn for these data set results on and are shown in figure 6. Areas under these curves are computed using Trapezoid rule and are shown in figure 7. Areas under these curves for MLP and Modular network models on data set DS5 are 0.634 and 0.655 respectively while area under curve for GFNN is 0.505 on data set DS4.

\section{CONCLUSION}

This paper proposes an effective ANN based approach for cardiac arrhythmia classification from standard 12 lead ECG signal recordings data. From the comparative analysis of results obtained, it is clear that the estimated MLP based ANN model with error back propagation algorithm can operate as an excellent classifier for the given cardiac arrhythmia classification task. We got classification accuracy of $\mathbf{8 6 . 6 7}$ and sensitivity of $\mathbf{9 3 . 7 5}$ on data set DS5 on MLP neural network model with two numbers of hidden layers which is much better than classification accuracy reported on the same problem. Classification results also shows that only one performance measure is not sufficient to measure classification performance of an ECG arrhythmia classifier. Experimental results shows that data set DS4 have given better classification performance on GFFNN model while data set DS5 have given best classification results on MLP and Modular NNs models with two numbers of hidden layers. MLP neural network model have given very attractive cardiac arrhythmia classification results on testing data.

We conclude that the system can be further developed and fine tuned for practical applications. Our future scope of research work will be performing experiments to classify different arrhythmia types instead of only binary classification from ECG signal recordings data and exploring the sensitivity of ECG features contributing to various arrhythmia types.

\section{REFERENCES}

[1] Dale Dubin , MD , "Rapid Interpretation of EKG's", USA, ISBN: 97809129120662001

[2] Zuo, W.M.; Lu, W.G.; Wang, K.Q.; Zhang, H., "Diagnosis of cardiac arrhythmia using kernel difference weighted KNN classifier", Computers in Cardiology 2008 , Page(s): 253 - 256

[3] G. D. Srinivasan N, Krishnan S. M., "Cardiac arrhythmia classification using autoregressive modeling", BioMed Eng Online, 2002, 1(1):5

[4] Pooja Bhardwaj, Rahul R Choudhary and Ravindra Dayama. Article: Analysis and Classification of Cardiac Arrhythmia Using ECG Signals. International Journal of Computer Applications 38(1):37-40, January 2012. Published by Foundation of Computer Science, New York, USA

[5] Raut, R.D.; Dudul, S.V., "Arrhythmias Classification with MLP Neural Network and Statistical Analysis", First IEEE International Conference on Emerging Trends in Engineering and Technology, 2008, Page(s): $553-$ 558

[6] G.Selvakumar, K.Boopathy Bagan, "Wavelet Decomposition for Detection and Classification of Critical ECG Arrhythmias", Proceeding of the 8th WSEAS International Conference on Mathematics and computers in Biology and Chemistry, June 2007, pp. 8084.

[7] G.Selvakumar, K.Boopathy Bagan, "An Efficient QRS Complex Detection Algorithm using Optimal Wavelet", WSEAS Transactions on Signal Processing, Volume 2, Issue 8, August 2006, pp. 1069-1073.

[8] Sung-Nien Yu,Ying-Hsiang Chen, "Electrocardiogram beat classification based on wavelet transformation and probabilistic neural network", Periodical Journal Pattern Recognition Letters, Elsevier Science Inc New York, NY, USA, Volume 28 , Issue 10, July 2007, pp. 11421150.

[9] Sung-Nien Yu,Ying-Hsiang Chen, "Electrocardiogram beat classification based on wavelet transformation and probabilistic neural network", periodical journal Pattern Recognition Letters, Elsevier Science Inc New York, NY, USA, Volume 28 , Issue 10, July 2007, pp. 11421150 . 
[10] Hafizah Hussain and Lai Len Fatt, "Efficient ECG Signal Classification Using Sparsely Connected Radial Basis Function Neural Network", Proceeding of the 6th WSEAS International Conference on Circuits, Systems, Electronics, Control and Signal Processing, December 2007, pp. 412-416.

[11] Rahime, Ceylan, Yuksel and Ozbay, "Comparison of FCM, PCA and WT techniques for classification ECG arrhythmias using artificial neural network", International Journal on Expert Systems with Applications Volume 33, Issue 2, August 2007, pp. 286295.

[12] Labib Khadra, Amjed S. Al-Fahoum, and Saed Binajjaj, "A quantitative analysis approach for cardiac arrhythmia classification using higher order spectral techniques", IEEE Transactions on Biomedical Engineering, Vol. 52, No. 11, November 2005, pp. 1840-1845

[13] Yang Wang, Yi.- Sheng Zhu, Nitish V. Thakor, and YuHong $\mathrm{Xu}$, "A Short Time Multifractal Approach for Arrhythmia Detection Based on Fuzzy Neural Network", IEEE Transactions on Biomedical Engineering, Vol. 48, No. 9, 2001, pp. 989-995.

[14] Shivajirao Jadhav, Sanjay Nalbalwar and Ashok Ghatol, "Modular Neural Network based Arrhythmia Classification System using ECG Signal Data", International Journal of Information Technology \& Knowledge Management (ISSN 0973-4414), JanuaryJune 2011, Vol. IV, No.:1 pp. 205-209

[15] Shivajirao Jadhav, Sanjay Nalbalwar and Ashok Ghatol, "Artificial Neural Network Based Cardiac Arrhythmia Classification Using ECG Signal Data", in Proc. Int. Conference on Electronics and Information Engineering, Kyoto Japan, Volume: 1, 10.1109/ICEIE.2010.5559887, Pages: V1-228 - 231

[16] Shivajirao Jadhav, Sanjay Nalbalwar and Ashok Ghatol, "ECG Arrhythmia Classification using Modular Neural Network Model", in Proc. 2010 IEEE EMBS Conference on Biomedical Engineering \& Sciences , IECBES.2010.5742200, Kuala Lumpur, Malaysia, Pages: 62-66

[17] Shivajirao Jadhav, Sanjay Nalbalwar and Ashok Ghatol, "Generalized Feedforward Neural Network based Cardiac Arrhythmia Classification from ECG Signal Data", 2010 6th International Conference on Advanced Information Management and Service (IMS) with ICMIA 2010, Seoul South Korea, Pages: 351-356

[18] Kemal Polat, Seral Şahan, Salih Güneş, A new method to medical diagnosis: Artificial immune recognition system (AIRS) with fuzzy weighted pre-processing and application to ECG arrhythmia, Expert Systems with Applications, Vol. 31, Issue 2, August 2006, pp. 264-269
[19] Jadhav, S.M.; Nalbalwar, S.L.; Ghatol, A.A.; "Arrhythmia disease classification using Artificial Neural Network model," Computational Intelligence and Computing Research (ICCIC), 2010 IEEE International Conference on , vol., no., pp.1-4, 28-29 Dec. 2010

[20] Jadhav, S.; Nalbalwar, S.; Ghatol, A., "Modular neural network model based foetal state classification", 2011 IEEE International Conference on Bioinformatics and Biomedicine Workshops (BIBMW 2011), pp.915-917

[21] Sang-Hong Lee, Jung-Kwon Uhm, and Joon S. Lim, "Extracting Input Features and Fuzzy Rules for Detecting ECG Arrhythmia Based on NEWFM", International Conference on Intelligent and Advanced Systems, Division of Software, Kyungwon University, Korea

[22] Alaa M. Elsayad, " Classification of ECG arrhythmia Using Learning Vector Quantization Neural Networks" (978-1-4244-5844-8/09/\$26.00 @2009 IEEE )Manuscript received July 30, 2009: revised 1 October 2010

[23] Uyar A., Gurgen F., "Arrhythmia Classification Using Serial Fusion of Support Vector Machines and Logistic Regression," Intelligent Data Acquisition and Advanced Computing Systems: Technology and Applications, 2007. IDAACS 2007. 4th IEEE Workshop on , vol., no., pp.560-565, 6-8 Sept. 2007

[24] Ali Mirza Mahmood and Mrithyumjaya Rao Kuppa, "A novel pruning approach using expert knowledge for dataspecific pruning", Engineering with Computers 28, 1 (January 2012), 21-30.

[25] Blake CL, Merz CJ. UCI Repository of Machine Learning Databases. 1998. Available from: http://archive.ics.uci.edu/ml/datasets.html (Downloaded Date: 25th January, 2012 )

[26] Lippman R., "An introduction to computing with neural nets", IEEE Trans. ASSP Magazine 4, 4-22,1987

[27] Jose Principe, Neil Euliano,Curt Lefebvre, Neural And Adaptive System, 2000 Jon Willey and Sons, Inc., New York

[28] Thomas J. Downey, Donald J. Meyer, Rumi Kato Price, Using the Receiver Operating Characteristic to assess the performance of Neural Classifiers, IEEE $1999,3642-3646$

[29] Fawcett T., ROC Graphs: Note and Practical Considerations for Data Mining Researchers, HP Labs Technical Report (HPL-2003-4), 2003

[Online]. (Downloaded date: 25 February 2012). Available: http://www.neurosolutions.com/download.h 